\title{
Walking pathways in cancer
}

Ph. Stegmaier ${ }^{1}$, O.V. Kel-Margoulis ${ }^{1}$, E. Wingender ${ }^{1,2}$, F.A. Kolpakov ${ }^{3}$, A.E. Kel ${ }^{1,4 *}$ 'geneXplain GmbH, Wolfenbuettel, Germany

${ }^{2}$ Institute of Bioinformatics, Goettingen University, Goettingen, Germany

${ }^{3}$ Biosoft.ru, Novosibirsk, Moscow, Russia

${ }^{4}$ Institute of Chemical Biology and Fundamental Medicine SB RAS, Novosibirsk, Russia

*e-mail:alexander.kel@genexplain.com

Key words: promoters, signal transduction, genomics, transcriptomics, cancer, TRANSFAC

Motivation and Aim: Regions of non-coding DNA in genomes are the source of high adaptability of molecular genomic systems of multicellular eukaryotic organisms (such as human) to varying external conditions. Such high adaptability is provided and maintained, first of all, through structural plasticity of gene regulatory networks. Binding of highly variable complexes of transcription factors to the fluctuating opened chromatin regions in genome (due to non-coding genomic and epigenomic variations) underlies the fundamental basis for such structural plasticity of gene regulatory networks. One of the most important results of the plasticity of the cancer pathways is the acquired resistance of tumors to chemotherapy. In this talk we will describe our results of analysis of noncoding regions of differentially expressed genes using TRANSFAC database can help to elucidate molecular mechanisms of cancer drug resistance and identify new potential therapeutic targets.

Methods and Algorithms: Empirical information about the interaction of transcription factors and the regulated target genes, obtained by either conventional or highthroughput methods, has been collected in the TRANSFAC database since 30 years. We used TRANSFAC to predict transcription factors (TFs) that regulate drug resistance genes in cancer. Analysis of signaling pathways that control the activity of TFs signal transduction network was done using most comprehensive signal transduction database TRANSPATH. With the help of geneXplain platform (http://www.genexplain.com) we identified structural changes in regulatory pathways with positive feedback loops, which helps to decipher the molecular mechanisms of the emergence of drug resistance and reveal potential drug targets .

Results: We analysed multiple "-omics" data in the cancer cells resistant to chemotherapy by methotrexate (MTX), including RNA-seq, ChiP-seq and phosphoproteomics. We identified the following potential drug targets against induced resistance of cancer cells: TGFalpha, IGFBP7, alpha9-integrin. Application of chemoinformatics tool PASS to revealed targets helped to identify the following chemical compounds: zardaverine, divalproex and human metabolite nicotinamide $\mathrm{N}$-oxide that potentially may be used to sensitization of cancer cells against MTX.

Conclusion: We think, that often non-reversible structural changes of the regulatory networks due to an epigenomic "evolution" of gene regulatory regions cause transformations in the system switching to a disease state. We call such structural network changes as "walking pathways". The analysis of this phenomenon helps us to to identify prospective drug targets to treat cancer.

Acknowledgements: The research has been supported in part by FASIE grant MirCol No. 340ГР/24467. 